\title{
LA INVESTIGACIÓN CIENTÍFICA EN LA FACULTAD DE CIENCIAS SOCIALES DE LA UNIVERSIDAD DE COSTA RICA
}

\section{SCIENTIFIC RESEARCH IN THE FACULTY OF SOCIAL SCIENCES OF THE UNIVERSITY OF COSTA RICA}

Daniel Camacho Monge

Director

\author{
PALABRAS CLAVE: COSTA RICA * INVESTIGACIÓN * CIENCIAS \\ SOCIALES * UNIVERSIDAD * DIVULGACIÓN CIENTÍFICA \\ KEYWORDS: COSTA RICA * RESEARCH * SOCIAL SCIENCES * \\ UNIVERSITIES * SCIENCE POPULARIZATION
}

En 1974, en cumplimiento de resoluciones del célebre Tercer Congreso Universitario (1972-1974) fue constituida la Facultad de Ciencias Sociales de la Universidad de Costa Rica para lo cual se reunieron varias unidades académicas - escuelas, institutos, secciones y departamentos - algunas de ellas de larga historia y tradición, que se encontraban diseminadas en distintos espacios de la Universidad. Las Segundas Jornadas de Investigación se realizaron entre el 22 al 25 de abril de 2019, año en el cual la Facultad llegó a sus cuarenta y cinco años de existencia. Aunque no es mi costumbre hacer referencias personales en la presentación que redacto para cada número de esta Revista, hago esta vez una excepción en vista del papel que me tocó ejercer como Secretario General del Tercer Congreso Universitario, del cual surge la Facultad, así como de mi desempeño en el cargo de primer Decano y el consiguiente reto de organizar la naciente institución.

Desde su nacimiento, la Facultad de Ciencias Sociales ha sido una de las más grandes de la Universidad por el número de estudiantes, escuelas y carreras y así se ha mantenido durante sus cuarenta y cinco años de existencia. Desde su inicio otorgaba grados en Antropología, Ciencias Políticas, Comunicación Colectiva, Geografía, Historia, Psicología, Sociología y Trabajo Social y realizaba investigaciones en esos campos, tanto en las escuelas como en dos institutos, el de Investigaciones Psicológicas, preexistente y el Instituto de Investigaciones Sociales que fundé durante mi periodo de Decano. Además, la Facultad intensificó las relaciones con la sociedad dentro del nuevo concepto de Acción Social, en consonancia con los lineamientos surgidos del Tercer Congreso.

Hoy en día se mantienen pujantes los mencionados institutos; además, tal como lo resalta páginas adelante el ex decano doctor Manuel Martínez, se han agregado el Centro de Investigaciones Antropológicas (CIAN), el Centro de Investigaciones en Comunicación (сісом), el Centro de Investigaciones y Estudios Políticos (CieP) y 
el Centro de Investigaciones Históricas de América Central (CIHAC). También en estos cuarenta y cinco años, la Facultad ha puesto en marcha una oferta de estudios de posgrado - maestrías y doctoradosimpresionante por su cantidad y calidad, punto al que también hace referencia el doctor Martínez.

También impresiona la intensa y numerosa participación en las Segundas Jornadas de Investigación donde se presentaron informes sobre ochenta investigaciones en curso y se dictaron más de cien conferencias. El material que esta revista presenta a continuación es solo una pequeña parte de ese acervo aportado por quienes aceptaron convertir sus exposiciones orales a formato de artículo científico. Sus contribuciones se refieren a temas como desigualdad social, protestas y movimientos sociales, salud, religión y política, comunicación, ambiente e historia.

En el eje de "Desigualdades Sociales", Angulo expone la cuestión social del trabajo a partir del pensamiento de Edlene Pimentel, quien discute las bases del capitalismo desde la pobreza y el desempleo. Asimismo, se analizan las posturas de los autores franceses Castel y Rosanvallon, a partir de su propuesta reformista ante la crisis de 1970. De esta manera, se estudian las posibilidades y los límites de esas diferentes posturas, con el fin de aportar al debate desde diversos postulados teóricos y acercarse a las interpretaciones de la crisis estructural del capital.

En esta misma línea, Fallas presenta una reflexión histórica sobre la crisis estructural del capital desde los años 70 y la crisis del Estado de Bienestar; señala la necesidad constante de expansión del capitalismo, basada en la acumulación y la caracterización de la fuerza de trabajo como mercancía. Comprender la dinámica histórica de estas etapas permite profundizar en el entendimiento de las luchas sociales, los procesos involucrados y los límites del capital.

En el eje de "Movimientos sociales y participación política", utilizando la base de datos denominada PROTESTAS del Instituto de Investigaciones Sociales de la Universidad de Costa Rica, Alvarado y Martínez presentan un monitoreo de las protestas durante la administración de Luis Guillermo Solís, utilizando la metodología de Análisis de Eventos de Protesta. De esta manera, se observó el uso de acciones de carácter institucional como no institucional, redefiniendo la dinámica entre el uso de ambos tipos de formatos. Una gran parte de estas demandas fueron de carácter salarial y de derechos laborales. Asimismo, se evidenciaron tres actores clave en este periodo: la población trabajadora, las madres, los padres y la población estudiantil, los vecinos y las vecinas. Entre los actores demandados se encuentra el poder ejecutivo, las instituciones autónomas y el poder legislativo. Este artículo es un buen aporte al estudio de los movimientos sociales, las tendencias, los tipos de demandas y las dinámicas de las protestas que se propiciaron en este determinado periodo de gobierno.

Mora hace un estudio sobre las demandas de los sindicatos de salud que se dieron a partir de la crisis de la Caja Costarricense del Seguro Social, entre los años 2012-2016. Para este estudio se revisaron los pronunciamientos de la Unión Nacional de Empleados de la Caja y la Seguridad Social (UnDECA) y del Sindicato de Profesionales de Ciencias 
Médicas de la Caja Costarricense de Seguro Social e Instituciones Afines (SIPROCIMECA). Asimismo, se revisaron informes relevantes emitidos por la Organización Panamericana de la Salud, la Defensoría de los Habitantes y la Asamblea Legislativa. Lo anterior permitió evidenciar como estas organizaciones constituyeron entes de denuncia, fiscalización y aportaron propuestas en defensa de la institucionalidad $y$ de los intereses de la población usuaria de los servicios de salud. Asimismo, se reflexiona sobre la impunidad presente en muchos casos de denuncia y delito dentro de esta institución.

En el eje "Política, poder y sociedad civil", Tapia analiza la coyuntura política que se dio en las elecciones del año 2018, cuando un grupo político-religioso irrumpió en la esfera social costarricense al presentar un alto grado de posibilidades de ocupar el poder ejecutivo. Por esta razón, se estudian las condiciones psicosociales y psicoreligiosas que llevaron a un sector de la población a emerger en el escenario político a partir de un grupo religioso. Se considera la teoría de la secularización para evidenciar una transformación constante e interminable, que ha puesto de manifiesto nuevas expresiones religiosas-espirituales con un origen social. Este estudio permite la reflexión hacia políticas que promuevan la inclusión social, la negociación y que eviten la autoexclusión.

Siguiendo esta temática, Garro y Solís realizan un estudio del sistema de medios costarricense, a nivel de ubicación geográfica, alcance y acceso al espectro radioelectrónico, a partir del principio de pluralidad. Asimismo, se caracterizaron 296 medios digitales, televisivos, radiofónicos e impresos, ubicándose la gran mayoría en la provincia de San José, lo cual genera procesos de concentración y exclusión. Otro punto relevante es el uso extendido del internet $y$ de las redes sociales en la actualidad. Lo anterior permite una reflexión sobre prácticas comunicativas, el ejercicio de expresión, el derecho a la información pública y la diversidad geográfica para su inclusión en las diferentes políticas públicas.

En el eje de "Feminismo, género y diversidades", Jiménez, Mena y Preinfalk realizan una investigación relacionada con la discriminación en el ámbito universitario, a partir de casos y percepciones de estudiantes de la Universidad Nacional de Costa Rica (UNA). Utiliza una metodología cualitativa por medio de grupos focales, revisión de normativa nacional e institucional, así como entrevistas al personal de la institución, entre el periodo 2017-2019. Se logró evidenciar situaciones de discriminación en diversas formas, ya sea por orientación sexual, discapacidad, origen étnico, identidad de género, religión o condición socioeconómica. La UNA es una institución que ha realizado esfuerzos por incluir sectores sociales con mayor desventaja social, así como velar por la prevención de la violencia y de la discriminación. Ha implementado varias normativas y reglamentos para brindar un espacio libre de discriminación. Por lo tanto, es relevante y de suma importancia fortalecer la inclusión de estos grupos en la toma de decisiones institucionales y brindar capacitación a toda la comunidad universitaria para eliminar este tipo de violencia en el estudiantado, con el fin de promover un compromiso político y ético de la población universitaria para garantizar el desarrollo social y profesional en la educación superior. 
En el eje de "Relación Sociedad/Naturaleza", Alfaro y Calvo presentan las principales demandas ambientales en la agenda pública y en las políticas de desarrollo en Costa Rica en el periodo 1995-2015, a partir del concepto de "cuestión ambiental" utilizado en la gestión de lo público. Para ello, se realizó una recopilación de noticias de diversos medios periodísticos, así como el estudio de diversos Planes de Desarrollo Nacional. A partir de lo anterior, se analizan las respuestas estatales a estas demandas, identificando ocho temáticas principales: agua, saneamiento, estudios acuíferos, cobertura forestal, impacto de monocultivos, energía, impacto de la minería y daño ambiental. Entre sus conclusiones se destacan la poca definición de roles y de funciones de las instancias públicas involucradas, adicional a una normativa desactualizada, así como el abordaje político-económico que se presenta en nuestro contexto.

Otro aporte en este tema es presentado por Blanco, al abordar el papel e impacto de los desarrollos turísticos en Guanacaste que se dieron a través de las políticas de impulso económico y turístico, con el fin de mejorar la calidad de vida de la población. El desarrollo turístico de esta zona se ha incrementado progresivamente a través de los años, por lo cual se evalúan indicadores de desempleo, pobreza y desigualdad, con el objetivo de determinar si este incremento en turismo se ha traducido en mejores condiciones de vida para sus habitantes. El Estado cumple un papel fundamental en esta situación, ya que su interés se ha centrado en atraer inversiones transnacionales, pero no brinda el apoyo necesario a los negocios locales para que puedan generar riqueza y mejorar su calidad de vida.

En en eje "Movimientos Sociales y participación política", Rojas hace un recorrido histórico de las huelgas realizadas por oficiantes de madera en el año 1918 en Costa Rica, con el fin de exigir mejores condiciones, siendo la más importante el establecimiento de jornadas laborales de ocho horas. Es relevante retomar este tipo de acontecimientos que marcaron un hecho destacado en la historia de los movimientos sociales y en la lucha por los derechos para la población trabajadora. 